KIAS-P98049

SNUTP 98-143

\title{
Four Light Neutrinos in Singular Seesaw Mechanism with Abelian Flavor Symmetry
}

\author{
Chun Liu ${ }^{a}$ and Jeonghyeon Song ${ }^{b}$ \\ ${ }^{a}$ Korea Institute for Advanced Study, 207-43 Chungryangri-dong, Dongdaemun-ku, \\ Seoul 130-012, Korea \\ ${ }^{b}$ Center for Theoretical Physics, Seoul National University, \\ Seoul 151-742, Korea
}

\begin{abstract}
The four light neutrino scenario, which explains the atmosphere, solar and LSND neutrino experiments, is studied in the framework of the seesaw mechanism. By taking both the Dirac and Majorana mass matrix of neutrinos to be singular, the four neutrino mass spectrum consisting of two almost degenerate pairs separated by a mass gap $\sim 1 \mathrm{eV}$ is naturally generated. Moreover the right-handed neutrino Majorana mass can be at $\sim 10^{14} \mathrm{GeV}$ scale unlike in the usual singular seesaw mechanism. Abelian flavor symmetry is used to produce the required neutrino mass pattern. A specific example of the flavor charge assignment is provided to show that maximal mixings between the $\nu_{\mu}-\nu_{\tau}$ and $\nu_{e}-\nu_{s}$ are respectively attributed to the atmosphere and solar neutrino anomalies while small mixing between two pairs to the LSND results. The implication in the other fermion masses is also discussed.
\end{abstract}

PACS numbers: $11.30 \mathrm{Hv}, 14.60 \mathrm{Pq}, 14.60 . \mathrm{St}$. 


\section{INTRODUCTION}

The recent Super-Kamiokande data on the zenith-angle-dependent deficit of atmospheric $\mu$-type neutrinos have provided compelling evidence of neutrino masses and mixing [1]. It might be the first discovery of physics beyond the standard model (SM), and has drawn a lot of theoretical attentions. The mass pattern of neutrinos is valuable information for the exploration of the physics related to the flavor puzzle in the SM. Theoretically, several mechanisms have been suggested to accommodate massive neutrinos. One of the most popular scenarios is the seesaw mechanism which naturally explains the smallness of neutrino mass by introducing heavy right-handed neutrinos [2]. Another example is the supersymmetric extension of the SM with R-parity violation. The trilinear lepton number violating interactions induce small neutrino masses at the loop level [3]. Since the whole flavor problem in the SM has not been understood yet, the detailed neutrino mass pattern in these models remains indefinite.

The observations of the atmospheric and solar neutrinos give the information about the neutrino mass squared differences and mixing angles under the assumption of neutrino oscillations. According to the Super-Kamiokande data, about $35 \%$ of the $\mu$-type atmospheric neutrinos change their flavor into non-e-type neutrinos, implying $\Delta m_{\mu x}^{2} \simeq 2.2 \times 10^{-3} \mathrm{eV}^{2}$ and $\sin ^{2} 2 \theta_{\mu x} \simeq 1(x \neq e)$ [1]. The solar neutrino deficit problem [4] can be explained by either the matter-enhanced oscillation (MSW effects) [5] or the vacuum oscillation [6]. The MSW solution allows two parameter spaces: $\Delta m_{\text {ey }}^{2} \simeq 5 \times 10^{-6} \mathrm{eV}^{2}$ with $\sin ^{2} 2 \theta_{\text {ey }} \simeq 6 \times 10^{-3}$, and $\Delta m_{e y}^{2} \simeq 2 \times 10^{-5} \mathrm{eV}^{2}$ with $\sin ^{2} 2 \theta_{e y} \simeq 0.8$. The vacuum oscillation solution is $\Delta m_{e y}^{2} \simeq 8 \times 10^{-11} \mathrm{eV}^{2}$ with $\sin ^{2} 2 \theta_{e y} \simeq 0.8$. If only the solar and atmospheric neutrino data are taken into account, they can be understood in the frameworks with three light neutrinos [7,8].

If we further consider the LSND experiment [9], however, something previously unexpected has to be introduced in the theory. When ascribing the detection of flavorchanging events to neutrino oscillations, the experiment indicates that $\Delta m_{e \mu}^{2} \simeq 1 \mathrm{eV}^{2}$ and 
$\sin ^{2}\left(2 \theta_{e \mu}\right) \simeq 10^{-2}$. To accommodate all the above experiments, three light neutrinos are not enough, at least one additional light neutrino $\nu_{s}$ is needed [10]. It should be sterile from the $Z^{0}$ decay. Even though the KARMEN [11] group has recently reported that large part of the favored parameter region of LSND is excluded, full confirmation of the LSND results still awaits future experiments. For example, a particular value of $\Delta m_{e \mu}^{2} \sim 6 \mathrm{eV}^{2}$ compatible with the LSND results does not contradict the KARMEN data since this $\Delta m^{2}$ is examined most sensitively at LSND while least sensitively at KARMEN. Moreover, neutrinos with mass scale of eV play an important role in understanding the dark matter problem. In astrophysics, it has been known that the Cold + Hot Dark Matter cosmological models (CHDM) agree best with the data on the cosmic microwave background anisotropies and the large-scale distribution of galaxies and clusters in the nearby universe [12]. With about $70 \%$ cold dark matter and about $10 \%$ baryonic matter, few-eV neutrino mass is requisite to account for the rest $20 \%$ hot dark matter.

In this paper, the four light neutrino scenario is adopted. Phenomenological studies [10] have shown that the following mass patterns are favored. In terms of the mass eigenstates, the four neutrinos are grouped into two pairs which are separated by a gap of $\sim 1 \mathrm{eV}$. The two neutrinos in a pair are almost degenerate compared to the gap. The atmosphere neutrino anomaly can be explained by any of two pairs, and the solar neutrino deficit by the other. The LSND data is explained by two neutrinos in different pairs. In terms of weak eigenstates, approximately speaking, $\nu_{\tau}$ can pair with either $\nu_{\mu}$ or $\nu_{e}$.

How to construct this spectrum of very small masses of four neutrinos is a theoretically challenging problem [13. One of the most appealing explanation for the smallness of neutrino masses is the seesaw mechanism which introduces three very heavy right-handed neutrinos $N_{\alpha(=e, \mu, \tau)}$ of Majorana masses $\sim M$. Moreover the generic presence of the $N_{\alpha(=e, \mu, \tau)}$ 's in many extensions of the SM like SO(10) GUT's and $\mathrm{E}_{6}$ string theories adds more charms to the mechanism. Since the ordinary seesaw mechanism predicts three light neutrinos and three very heavy neutrinos, however, there is no room for light sterile neutrinos. Even though the so-called singular seesaw mechanism, where the Majorana mass matrix of the 
$N_{\alpha(=e, \mu, \tau)}$ 's is singular, has been suggested for this problem, a drawback occurs such that the characteristic mass scale of lepton number violation is too low. It deserts one of the strongest merits of the seesaw mechanism itself.

In this letter we propose a four neutrino scenario in the framework of the seesaw mechanism, which maintains the $M$ at GUT scales so to appreciate all the original attractions of the seesaw mechanism, and naturally produces the mass spectrum such that two almost degenerate pairs are separated. In addition, the physical origin of our scenario in the view point of the Abelian flavor symmetry is also discussed through a specific example to explain all the three neutrino anomalies.

\section{A MODEL FOR FOUR LIGHT NEUTRINOS}

Natural generation of neutrino masses much lighter than the electroweak scale brings popularity to the seesaw mechanism. As introducing three right-handed neutrinos $N_{\alpha(=e, \mu, \tau)}$ with Majorana mass $\sim M$, ordinary seesaw mechanism have two mass scales, the heavy neutrino mass $M$ and the seesaw suppressed neutrino mass $m^{2} / M$, where the $m$ is the Dirac mass. Assigning the $m$ at the electroweak scale, the SM singlets $N_{\alpha(=e, \mu, \tau)}$ 's can have masses in the phenomenologically interesting range such that $M \sim 10^{13}-10^{16} \mathrm{GeV}$ corresponding to a light neutrino mass $m_{\nu} \sim 10^{-3}-1 \mathrm{eV}$. In order to accommodate three neutrino experiment results one of the right-handed neutrinos should be light. This requires the singular seesaw mechanism: the right-handed neutrino Majorana mass matrix is singular [14]. In Ref. [15], this mechanism was used to produce the four light neutrino mass pattern. In their approach, however, a drawback appears such that the mass of the heavy right-handed neutrinos is at $\mathrm{keV}$ scale, which is further explained by introducing double seesaw mechanism. This is because in Ref. [15 three mass scales, $M, m$, and $m^{2} / M$ are introduced. When the $m$ is charged with the LSND results and hot dark matter while the $m^{2} / M$ with the solar neutrino problem, the $M$ becomes much smaller than the GUT scale.

We observe that if the Dirac mass matrix is also singular, the right-handed neutrino mass 
can be pulled back to GUT scale or so even in singular seesaw mechanism. For illustrative purpose, the mass matrix of the following simple form is considered:

$$
\mathcal{M}=\left[\begin{array}{cccccc}
0 & 0 & 0 & 0 & 0 & 0 \\
0 & 0 & 0 & 0 & m_{22} & m_{23} \\
0 & 0 & 0 & 0 & m_{32} & m_{33} \\
0 & 0 & 0 & 0 & 0 & 0 \\
0 & m_{22} & m_{32} & 0 & M_{22} & M_{23} \\
0 & m_{23} & m_{33} & 0 & M_{23} & M_{33}
\end{array}\right],
$$

which is of rank four. In the mass spectrum, there are two heavy neutrinos of masses $\sim M$, two light neutrinos of masses $\sim m^{2} / M$, and two massless neutrinos. In other words, the three scales in this case are $M, m^{2} / M$, and 0 . The four light neutrinos are naturally divided into two pairs with a mass hierarchy of $\sim m^{2} / M$, and each pair consists of two degenerate mass eigenstates.

Thus the seesaw mechanism, under the assumption that both the Dirac and Majorana mass matrices are singular, can naturally produce the looking-bizarre but required mass pattern to explain the three neutrino anomalies. Moreover, the $M$ can be around $10^{13} \mathrm{GeV}$ if the $m$ is taken to be at the electroweak scale and the $m^{2} / M$ at $1 \mathrm{eV}$ scale, maintaining a merit of the seesaw mechanism such that it can be easily implemented in numerous theories for physics beyond the SM.

Now let us explore the physical origin of the singular seesaw mechanism, which could lead to the specific neutrino mass matrix texture in (1). It is natural to expect that there exists some symmetry to induce such neutrino mass pattern. This symmetry should also provide the large mixing for the atmosphere neutrino anomaly, which is no longer automatic in (1)

\footnotetext{
${ }^{1}$ Compared to the neutrino mass matrix which yields a degenerate neutrino pair with maximum mixing in the original singular seesaw mechanism [15], (1) gives vanishing mass to the two neutrinos. In our case, their mass eigenstates can be always rotated to the weak eigenstates.
} 
Furthermore, a softly breaking of the symmetry is necessary to generate small masses for two massless neutrinos and to lift the degeneracy in each pair.

It is known that Abelian flavor symmetry breaking by small parameters could be an answer of the hierarchy in the fermion masses [16]. We will use it to discuss the neutrino masses. In the following discussion, supersymmetry is implied. The flavor symmetry is spontaneously broken by a vacuum expectation value (VEV) of an electroweak singlet field $X$. As long as the flavor charges balance under the Abelian flavor symmetry, the following interactions are allowed:

$$
L_{\alpha} H N_{\beta}\left(\frac{\langle X\rangle_{\mathrm{VEV}}}{\Lambda}\right)^{m_{\alpha \beta}}, \quad M N_{\alpha} N_{\beta}\left(\frac{\langle X\rangle_{\mathrm{VEV}}}{\Lambda}\right)^{n_{\alpha \beta}}
$$

where $L_{\alpha}(\alpha=e, \mu, \tau), H$, and $N_{\alpha}$ denote the lepton doublets, one Higgs field, and the right-handed neutrino fields, respectively. The $\Lambda$ is the flavor symmetry breaking scale, and the condition $m_{\alpha \beta}, n_{\alpha \beta} \geq 0$ is required for the holomorphy of the superpotential. The order parameter for this new symmetry is defined by

$$
\lambda \equiv \frac{\langle X\rangle_{\mathrm{VEV}}}{\Lambda} \ll 1
$$

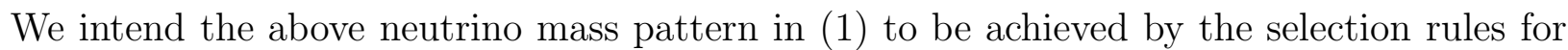
(2) through proper assignment of the flavor charges to $L_{\alpha}$ and $N_{\alpha}$. A further requirement is that the atmosphere neutrino anomaly is due to the $\nu_{\mu}-\nu_{\tau}$ oscillation. Compared to analogous analysis for three light neutrino scenario which does not count the LSND result [8], the choice of the flavor charges here is more limited. One tricky point is that one of the right-handed neutrino masses is made to be vanishingly small.

As a specific example, we consider the following assignment of the Abelian flavor charges:

$$
\begin{aligned}
& L_{e}(2 t-a), \quad L_{\mu}(a), \quad L_{\tau}(-a-2), \\
& E_{e}^{c}(-x), \quad E_{\mu}^{c}(-a+6), \quad E_{\tau}^{c}(a+6), \\
& N_{e}(2 r+a), \quad N_{\mu}(-a), \quad N_{\tau}(a+2), \quad X(-2),
\end{aligned}
$$

where the integers $a, t$, and $r$ are constrained as 


$$
1<t+1<a<x<r
$$

The $E_{\alpha}^{c}$ 's are the anti-particle fields of the $\mathrm{SU}(2)$ singlet charged leptons. In order to obtain the physical mixing angles of neutrinos, we should simultaneously take into account of the mass matrix for the charged lepton sector. The fields of gauge bosons and Higgs' possess vanishing flavor charges. It is to be noted that all the flavor charges for the second and third generations are expressed by a single parameter $a$.

The flavor charge assignment in (44) and (5) produces the Dirac and Majorana mass matrices of neutrinos as

$$
\mathcal{M}_{D}=m\left[\begin{array}{ccc}
Y_{11} \lambda^{r+t} & 0 & Y_{13} \lambda^{t+1} \\
Y_{21} \lambda^{r+a} & 1 & Y_{23} \lambda^{a+1} \\
Y_{31} \lambda^{r-1} & 0 & 1
\end{array}\right], \quad \mathcal{M}_{M}=\lambda M\left[\begin{array}{ccc}
\zeta_{1} \lambda^{2 r+a-1} & \zeta_{2} \lambda^{r-1} & \zeta_{3} \lambda^{r+a} \\
\zeta_{2} \lambda^{r-1} & 0 & 1 \\
\zeta_{3} \lambda^{r+a} & 1 & \zeta_{4} \lambda^{a+1}
\end{array}\right]
$$

and the mass matrix of the charged leptons as

$$
\mathcal{M}_{l}=m \lambda^{2}\left[\begin{array}{ccc}
0 & 0 & \eta_{13} \lambda^{t+1} \\
0 & \eta_{22} \lambda & \eta_{23} \lambda^{a+1} \\
0 & 0 & 1
\end{array}\right]
$$

where $Y$ 's, $\zeta$ 's and $\eta$ 's are order one coefficients. To the leading order, only tau lepton acquires mass $\lambda^{2} m$ while the muon and the electron remain massless. The mass matrix of four light neutrinos is obtained as follows, to the leading order,

$$
\mathcal{M}_{\nu}^{(0)} \simeq \frac{\epsilon^{2} M}{\lambda}\left[\begin{array}{cccc}
0 & 0 & 0 & 0 \\
0 & 0 & -1 & 0 \\
0 & -1 & 0 & 0 \\
0 & 0 & 0 & 0
\end{array}\right] \text {. }
$$

Here the $\epsilon$ denotes the ratio of the weak scale to the GUT scale, $\epsilon \equiv m / M$. The neutrino mass spectrum due to $\mathcal{M}_{\nu}^{(0)}$ is

$$
\mathcal{M}_{\nu}^{(0)} \Longrightarrow\left[m_{\nu_{1}}=m_{\nu_{2}}=0, \quad m_{\nu_{3}}=m_{\nu_{4}}=\frac{\epsilon^{2} M}{\lambda}, \quad \sin \theta_{34}=\frac{1}{\sqrt{2}}\right] \text {. }
$$


To attribute the LSND data to the oscillation between two groups, we require

$$
\frac{m^{2}}{\lambda M} \sim 1 \mathrm{eV}
$$

which can be satisfied with the following masses:

$$
m \simeq 10^{2} \mathrm{GeV}, \quad \lambda M \sim 10^{13} \mathrm{GeV} .
$$

One of the merits of our charge assignment is that we can obtain larger mass scale for $M$, according to the small value $\lambda$. If $\lambda \sim 10^{-1}$, which is a typical order of Cabbibo angle, the tau lepton mass is properly obtained:

$$
m_{\tau} \sim \lambda^{2} m \sim 1 \mathrm{GeV} .
$$

The full mass matrix of the charged leptons in (7) is solved by the standard method. The eigenvalues of the $\mathcal{M}_{l}$ are $0, \lambda^{3} m$, and $\lambda^{2} m$. In our mechanism with the flavor charge assignment in (4) and (5), the muon acquires the mass with appropriate order of magnitude $(\sim 100 \mathrm{MeV})$ but the electron is left massless even under the Abelian flavor symmetry breaking. The $\mathcal{M}_{l}$ is diagonalized by

$$
R_{l}^{L} \mathcal{M}_{l} R_{l}^{R \dagger}=\operatorname{Diag}\left(m_{e}, m_{\mu}, m_{\tau}\right)
$$

Since the $R_{l}^{L}$ diagonalizes the hermitian mass-squared matrix $\mathcal{M}_{l} \mathcal{M}_{l}^{\dagger}$, we have

$$
R_{l}^{L}=\left[\begin{array}{ccc}
1 & 0 & 0 \\
0 & 1 & -\eta_{23} \lambda^{a+1} \\
0 & \eta_{23} \lambda^{a+1} & 1
\end{array}\right] .
$$

In the neutrino sector the mass matrix of four light neutrinos can be obtained by the method described in Ref. [15]. The $\mathcal{M}_{M}$ is diagonalized to give three eigenvalues $\sim \lambda^{2 r+a} M, M$, and $-M$ by a rotation matrix $R_{M}$,

$$
R_{M}=\left[\begin{array}{ccc}
1 & 0 & -\zeta_{2} \lambda^{r-1} \\
\zeta_{2} \lambda^{r-1} / \sqrt{2} & 1 / \sqrt{2} & 1 / \sqrt{2} \\
\zeta_{2} \lambda^{r-1} / \sqrt{2} & -1 / \sqrt{2} & 1 / \sqrt{2}
\end{array}\right] .
$$


We finally have the symmetric mass matrix of four light neutrinos,

$$
\mathcal{M}_{\nu}=\frac{\epsilon^{2} M}{\lambda}\left[\begin{array}{cccc}
0 & \lambda^{t+1} & 0 & \lambda^{r+t+1} / \epsilon \\
\lambda^{t+1} & \lambda^{a} & -1 & \lambda^{r+a+1} / \epsilon \\
0 & -1 & \lambda^{a+1} & \lambda^{r} / \epsilon \\
\lambda^{r+t+1} / \epsilon & \lambda^{r+a+1} / \epsilon & \lambda^{r} / \epsilon & \lambda^{2 r+a+1} / \epsilon^{2}
\end{array}\right],
$$

where the charged lepton mixing effects are incorporated so that the charged lepton fields have been rotated to be mass eigenstates. In (16) each element denotes the order of magnitude estimate. Now the mixing angle for the LSND results comes from the $\lambda^{r} / \epsilon$ term, as

$$
\sin \theta_{\mathrm{LSND}} \sim \frac{\lambda^{r}}{\epsilon} \sim 3 \times 10^{-2}
$$

Since this mixing angle is small, the masses of $\nu_{3}$ and $\nu_{4}$ approximately come from (22), (23), and (33) components of $\mathcal{M}_{\nu}$ which implies the mass difference $\Delta m_{34} \sim \lambda^{a}$. The Super-Kamiokande data are explained as

$$
\Delta^{2} m_{34} \sim \lambda^{a}\left(\frac{m^{2}}{\lambda M}\right)^{2} \sim 10^{-3} \mathrm{eV}^{2}, \quad \text { for } a=3,
$$

where (10) has been used. Secondly, the masses of $\nu_{1}$ and $\nu_{2}$ approximately come from (11), (14), and (44) components of $\mathcal{M}_{\nu}$. Since $t<a$ in our charge assignment, we naturally have maximal mixing between $\nu_{1}$ and $\nu_{2}$ with the mass squared difference as

$$
\sin \theta_{12} \simeq \frac{1}{\sqrt{2}} \& \Delta m_{12}^{2} \sim 10^{-10} \mathrm{eV}^{2}, \text { for } t=1
$$

This corresponds to the vacuum oscillation solution for the solar neutrino problem.

Let us summarize the characteristic features of our mechanism:

- The four neutrino mass spectrum consisting of two almost degenerate pairs separated by a mass gap $\sim 1 \mathrm{eV}$ is naturally generated.

- The mass scale of the right-handed neutrinos in our case is high enough even in the singular seesaw mechanism. The understanding of neutrino mass and mixing can be put into the same category as the charged leptons and quarks. 
- The large mixing for the atmosphere neutrino problem is not automatic. It is achieved by introducing Abelian family symmetry. The $\nu_{\mu}-\nu_{\tau}$ large mixing is feasible in this case.

- The lightest neutrino masses are not generated by seesaw mechanism. The large mixing of the vacuum oscillation solution for the solar neutrino problem can be accommodated.

\section{DISCUSSION AND SUMMARY}

In this four light neutrino scenario, the flavor charge assignment or the texture of the neutrino mass matrix may imply that the first generation fermions are exceptional as far as their masses are concerned. For the second and third generations, neutrinos have been treated essentially the same as in three light neutrino scenarios using the ordinary seesaw mechanism [8]. Thus we expect that the charged lepton and quark masses of these two generations can be naturally understood within the framework of Abelian flavor symmetry. For the first generation, although it is possible to produce appropriate masses of the charged fermions by introducing some exotic flavor quantum numbers, the Yukawa couplings with flavor symmetry might not be the source for the masses. In the following, we point out a possibility of the mass origin of the first generation fermions which was mentioned in Ref. [17]. In the supersymmetric model with R-parity violation and baryon number conservation, the following interactions are allowed by the gauge symmetry and the flavor symmetry, with positive definite integers $m$ and $n$,

$$
L L E^{c}\left(\frac{\langle X\rangle_{\mathrm{VEV}}}{\Lambda}\right)^{m}, \quad Q L D^{c}\left(\frac{\langle X\rangle_{\mathrm{VEV}}}{\Lambda}\right)^{n}
$$

where we have suppressed the generation indices. The $Q$ and $D^{c}$ are the $\mathrm{SU}(2)$ quark doublet and down-type anti-quark singlet superfields, respectively. We note that if the sneutrino fields get non-vanishing VEVs, the above interactions generate masses for the charged leptons and down-type quarks. When the sneutrino VEVs are around $\sim\left(10^{-3}-10\right) \mathrm{GeV}$, the 
correct magnitudes for electron and down quark masses can be obtained[2]. One interesting point is that the up quark remains massless, so that there is no strong CP problem [19. Recently a similar idea was carried out in detail in Ref. 20.

In summary, within the framework of singular seesaw mechanism, we have studied the four light neutrino scenario which explains the atmosphere, solar, and LSND neutrino experiments. By taking the Dirac neutrino mass matrix to be also singular, the right-handed neutrino Majorana mass can be at $\sim 10^{14} \mathrm{GeV}$ scale. Abelian flavor symmetry is used to produce the required neutrino mass pattern.

\section{ACKNOWLEDGMENTS}

We would like to thank E.J. Chun, C. Giunti, S.K. Kang and C.W. Kim for various helpful discussions. J.S. is supported in part by KOSEF through CTP, SNU.

\footnotetext{
${ }^{2} \mathrm{~A} \mathrm{GeV}$ sneutrino VEV can result in a $\mathrm{MeV} \tau$ neutrino mass at tree level in general. However this conclusion is model-dependent. For example, the model described in Ref. [18] has vanishing $\tau$ neutrino mass at tree level.
} 


\section{REFERENCES}

[1] Super-Kamiokande Collaboration, Y. Fukuda et al., Phys. Rev. Lett. 81 (1998) 1562; T. Kajita, talk at Neutrino '98 (Takayama, June 1998).

[2] M. Gell-Mann, P. Ramond and R. Slansky, in Sanibel talk, CALT-68-709, Feb. 1979 (unpublished), and in Supergravity (North Holland, Amsterdam, 1979); T. Yanagida, in Proc. of the Workshop on Unified Theory and Baryon Number of the Universe (KEK, 1979).

[3] C. Aulakh and R. Mohapatra, Phys. Lett. B119 (1982) 136; S. Dimpoulos and L. Hall, Phys. Lett B207 (1988) 210; C. Liu, Mod. Phys. Lett. A 12 (1997) 329; M. Drees, S. Pakvasa, X. Tata and T. Veldhuis, Phys. Rev. D 57 (1998) R5335; E.J. Chun, S.K. Kang, C.W. Kim and U.W. Lee, hep-ph/9807327.

[4] J.N. Bahcall, P.I. Krastev, and A. Yu. Smirnov, Phys. Rev. D 58 (1998) 096016.

[5] S.P. Mikheyev and A. Yu Smirnov, Sov. J. Nucl. Phys. 42 (1985) 913; L. Wolfenstein, Phys. Rev. D 17 (1978) 2369.

[6] For examples, see V. Barger, R.J.N. Phillips and K. Whisnant, Phys. Rev. Lett. 69 (1992) 3135; S.L. Glashow, P.J. Kernan, and L.M. Krauss, hep-ph/9808470.

[7] For examples, see J.A. Harvey, P. Ramond and D.B. Reiss, Nucl. Phys. B199 (1982) 223; M. Bando, T. Kugo and K. Yoshioka, Phys. Rev. Lett. 80 (1998) 3004; C.H. Albright, K.S. Babu and S.M. Barr, Phys. Rev. Lett. 81 (1998) 1167; B. Brahmachari and R.N. Mohapatra, Phys. Rev. D 58 (1998) 015001; C. Giunti, C.W. Kim and M. Monteno, Nucl. Phys. B521 (1998) 3; F. Vissani, hep-ph/9708483; H. Fritzsch and Z.Z. Xing, Phys. Lett. B440 (1998) 313; E. Ma, D.P. Roy and U. Sarkar, hep-ph/9810309; Y.-L. Wu, hep-ph/9810491; K. Choi, K. Hwang and E.J. Chun, hep-ph/9811363.

[8] Y. Grossman and Y. Nir, Nucl. Phys. B448 (1995) 30; P. Binétruy et al., Nucl. Phys. B496 (1997) 3; N. Irges, S. Lavignac and P. Ramond, Phys. Rev. D 58 (1998) 035003; 
B.C. Allanach, hep-ph/9806294; J.K. Elwood, N. Irges and P. Ramond, Phys. Rev. Lett. 81 (1998) 5064; Y. Grossman, Y. Nir and Y. Shadmi, J. High Energy Phys. 10 (1998) 007; W. Buchmüller and T. Yanagida, hep-ph/9810308.

[9] LSND Collaboration, C. Athanassopoulos et al., Phys. Rev. Lett. 75 (1995) 2650.

[10] D.O. Caldwell and R.N. Mohapatra, Phys. Rev. D 48 (1993) 3259; J.T. Peltoniemi and J.W.F. Valle, Nucl. Phys. B406 (1993) 409; R.Foot and R.R. Volkas, Phys. Rev. D 52 (1995) 6595; E. Ma and P. Roy, Phys. Rev. D 52 (1995) R4780; E.J. Chun, A.S. Joshipura, A.Yu. Smirnov, Phys. Lett. B357 (1995) 371; J.J. Gomez-Cadenas and M.C. Gonzales-Garcia, Z. Phys. C71 (1996) 443; N. Okada and O.Yasuda, Int. J. Mod. Phys. A 12 (1997) 3669; S.M. Bilenky, C. Giunti and W. Grimus, Eur. Phys. J. C1 (1998) 247; Q.Y. Liu and A. Yu Smirnov, Nucl Phys. B524 (1998) 505; V.Barger, S. Pakvasa, T.J. Weiler and K. Whisnant, Phys. Rev. D58 (1998) 093016; Q. Shafi and Z. Tavartkiladze, hep-ph/9811463.

[11] B. Zeitnits, talk at Neutrino '98 (Takayama, June 1998).

[12] For a review, see J.R. Primack, astro-ph/9707285, to appear in the Proc. of Midrasha Mathematicae in Jerusalem: Winter School in Dynamical Systems (Jerusalem, Jan. 1997); J.R. Primack and M.A.K. Gross, astro-ph/9810204; J.R. Primack, J. Holtzman, A. Klypin, and D.O. Coldwel, Phys. Rev. Lett. 74 (1995) 2160.

[13] The extension of the radiative Zee model with the gauge group $\mathrm{SU}(2)_{L} \times \mathrm{U}(1)_{Y} \times \mathrm{U}^{\prime}(1)$, a $Z_{2}$ discrete symmetry, and hierarchical Yukawa couplings is used to include a fourth sterile neutrino, see N. Gaur, A. Ghosal, E. Ma and P.Roy, Phys. Rev. D58 (1998) 071301; P. Roy, hep-ph/9810448.

[14] R. Johnson, S. Ranfone and J. Schechfer, Phys. Lett. B179 (1986) 355; S.L. Glashow, Phys. Lett. B256 (1991) 255; M. Fukugita and T. Yanagida, Phys. Rev. Lett. 66 (1991) 2705; D. Du and C. Liu, Commun. Theor. Phys. 19 (1993) 117. 
[15] E.J. Chun, C.W. Kim and U.W. Lee, Phys. Rev. D 58 (1998) 093003; Y. Chikira, N. Haba and Y. Mimura, hep-ph/9808254.

[16] C.D. Frogatt and H.B. Nielsen, Nucl. Phys. B147 (1979) 277.

[17] C. Liu, Int. J. Mod. Phys. A 11 (1996) 4307.

[18] C. Liu, in Ref. [3].

[19] K. Choi, C. W. Kim and W. K. Sze, Phys. Rev. Lett. 61, 794 (1988); K. Choi, Nucl. Phys. B383, 58 (1992).

[20] J.E. Kim, hep-ph/9811509; J.E. Kim, B. Kyae and J.S. Lee, hep-ph/9811510. 